\title{
Rank Functions of Fuzzy Greedoids
}

\author{
Steven J. Tedford \\ Department of Mathematics and Computer Science, Misericordia University, Dallas, USA \\ Email: stedford@misericordia.edu
}

Received 19 February 2015; accepted 9 August 2015; published 12 August 2015

Copyright (C) 2015 by author and Scientific Research Publishing Inc.

This work is licensed under the Creative Commons Attribution International License (CC BY).

http://creativecommons.org/licenses/by/4.0/

(c) () Open Access

\begin{abstract}
Fuzzy greedoids were recently introduced as a fuzzy set generalization of (crisp) greedoids. We characterize fuzzy languages which define fuzzy greedoids, give necessary properties and sufficient properties of the fuzzy rank function of a fuzzy greedoid, give a characterization of the rank function for a weighted greedoid, and discuss the rank closure of a fuzzy greedoid.
\end{abstract}

\section{Keywords}

Fuzzy System Models, Greedoids, Fuzzy Rank Function

\section{Introduction}

In 1965 Zadeh [1] introduced the ideas of fuzzy set theory to study problems where partial membership in a set was appropriate. Since that time, work has progressed in extending his ideas to other areas of mathematics including analysis, linear algebra, and combinatorics.

In fact, starting in 1988, Goetschel Jr. and Voxman introduced the idea of a fuzzy matroid through a series of papers studying the many different cryptomorphic definitions of a crisp matroid [2]-[4].

Only recently, in 2011, Al-Hawary [5] applied fuzzy set theory to the more general set system of a greedoid. Greedoids generalize both matroids and antimatroids, along with many other set systems. They arose from the study of the greedy algorithm and optimization [6]. See [7] for an thorough introduction to the study of greedoids.

Similar to matroids and other set systems, greedoids can be defined in a variety of ways, including as a set system, as an ordered language, from a rank function, and from a "closure operator". In this article, we consider some of the other ways that fuzzy greedoids may be defined. We obtain a characterization of fuzzy greedoids in terms of fuzzy languages, and obtain necessary conditions and also sufficient conditions for a function to be a rank function of a fuzzy greedoids. Finally, we discuss the existence of a closure operator for a fuzzy greedoid.

In Section 2, we state the basic definitions and give a large class of examples of fuzzy greedoids. In Section 3, we introduce the ordered language interpretation of fuzzy greedoids. In the fourth section we discuss the rank 
function, introducing both sufficiently and separately necessary conditions for the rank function of general fuzzy greedoids. Additionally we obtain a characterization for the rank function of weighted greedoids (a large class of fuzzy greedoids). In Section 5 we discuss the existence of a rank closure operator in fuzzy greedoids. We finish with some open areas of research relating to fuzzy greedoids.

\section{Definitions and Examples}

Although it is possible to define fuzzy sets on any set, we are going to assume that the underlying set $E$ is finite. A fuzzy set on $E$ is a function $\mu: E \rightarrow[0,1]$. This generalizes the idea of a subset, which can be thought of as a function from $E$ into $\{0,1\}$. For each $e \in E, \mu(e)$ is call the height of $\mu$ at $e$. Similar to (crisp) set theory, there are some standard definitions and operations for fuzzy sets. We list these definitions below, with their corresponding names.

Definition 1. Suppose that $\mu, v$ are fuzzy sets on $E$ and $e \in E$. Then

1) $(\mu \vee v)(e)=\max \{\mu(e), v(e)\} \quad$ (fuzzy union);

2) $(\mu \wedge v)(e)=\min \{\mu(e), v(e)\} \quad$ (fuzzy intersection);

3) $(\mu-v)(e)=\min \{\mu(e), 1-v(e)\}$ (fuzzy set difference);

4) $\operatorname{supp}(\mu)=\{e \in E \mid \mu(e)>0\} \quad$ (support of $\mu$ );

5) $m(\mu)=\min \{\mu(e) \mid e \in \operatorname{supp}(\mu)\} \quad$ (minimal height);

6) $\mu \leq v$ if and only if $\mu(e) \leq v(e)$ for all $e \in E$;

7) $\mu<v$ if and only if $\mu \leq v$ and there exist $e \in E$ such that $\mu(e)<v(e)$;

8) $|\mu|=\sum_{e \in \sup (\mu)} \mu(e)$.

Finally, we let $[0,1]^{E}$ be the set of all fuzzy sets on $E$.

There is one particular type of fuzzy set which we will use extensively throughout the article-a spikewhich we define here:

Definition 2. Suppose $e \in E$ and $h \in(0,1]$. We define a spike $\sigma_{e}^{h}$ to be the fuzzy set whose support is e and whose height at e is $h$.

Every fuzzy set can be thought of as a fuzzy union of spikes, as the following lemma, whose proof is straightforward, shows.

Lemma 3. Suppose $\mu \in[0,1]^{E}$ with $\operatorname{supp}(\mu)=\left\{e_{1}, \cdots, e_{k}\right\}$. If $\mu\left(e_{i}\right)=h_{i}$, then $\mu=\sigma_{e_{1}}^{h_{1}} \vee \sigma_{e_{2}}^{h_{2}} \vee \cdots \vee \sigma_{e_{k}}^{h_{k}}$.

We now want to generalize the definition of a (crisp) greedoid to a fuzzy set version. The traditional definition of a greedoid in term of its feasible sets is given below. A thorough introduction to crisp greedoid theory can be found in [7].

Definition 4. A finite set $E$ and a collection $\mathcal{F}$ of subsets of $E$ forms a (crisp) greedoid if and only if

1) $\varnothing \in \mathcal{F}$;

2) For any $F \in \mathcal{F}$ with $F \neq \varnothing$, there exists $e \in F$ such that $F-e \in \mathcal{F}$;

3) If $F, F^{\prime} \in \mathcal{F}$ with $|F|<\left|F^{\prime}\right|$ then there exists $e \in F^{\prime}-F$ such that $F \cup e \in \mathcal{F}$.

By considering this definition in terms of functions from $E$ to $[0,1]$, we can extend the definition of a greedoid to a fuzzy greedoid. We will use the definition given by Al-Hawary [5].

Definition 5. Given a finite set $E$ and a family $\mathcal{F}_{f} \subseteq[0,1]^{E}$ of fuzzy sets satisfying the following two conditions:

(F1) For every $\mu \in \mathcal{F}_{f}$ with $\mu \neq 0^{E}$, there exists $x \in \operatorname{supp}(\mu)$ such that $\mu-\sigma_{x}^{\mu(x)} \in \mathcal{F}_{f}$;

(F2) For any $\mu_{1}, \mu_{2} \in \mathcal{F}_{f}$ with $0 \leq\left|\operatorname{supp}\left(\mu_{1}\right)\right|<\left|\operatorname{supp}\left(\mu_{2}\right)\right|$, then there exist $\mu \in \mathcal{F}_{f}$ such that:

1) $\mu_{1} \leq \mu \leq \mu_{1} \vee \mu_{2}$;

2) $m(\mu) \geq \min \left\{m\left(\mu_{1}\right), m\left(\mu_{2}\right)\right\}$. 
Then the fuzzy set system $\mathfrak{G}=\left(E, \mathcal{F}_{f}\right)$ is called a fuzzy greedoid and the elements of $\mathcal{F}_{f}$ are the feasible fuzzy sets of $\mathfrak{G}$.

Because of their close relationship with crisp greedoids, a large number of examples of fuzzy greedoids can be obtained by adding a weight function to a greedoid, as the following proposition shows.

Proposition 6. Suppose $G=(E, \mathcal{F})$ is a crisp greedoid. Let $w: E \rightarrow[0,1]$ be a weight function on $E$. If

$$
\mathcal{F}_{f}=\left\{\mu \in[0,1]^{E} \mid \operatorname{supp}(\mu) \in \mathcal{F} \text { and } \forall e \in \operatorname{supp}(\mu), \mu(e)=w(e)\right\},
$$

then $\mathfrak{G}=\left(E, \mathcal{F}_{f}\right)$ is a fuzzy greedoid.

Proof. All that is required is to show that $\mathcal{F}_{f}$ satisfies both (F1) and (F2). Suppose $\mu \in \mathcal{F}_{f}$. Since $\operatorname{supp}(\mu) \in \mathcal{F}$, there exist $x \in \operatorname{supp}(\mu)$ such that $\operatorname{supp}(\mu)-x \in \mathcal{F}$. Thus $\mu-\sigma_{x}^{w(x)} \in \mathcal{F}_{f}$. This implies that $\mathcal{F}_{f}$ satisfies (F1).

Suppose $\mu_{1}, \mu_{2} \in \mathcal{F}_{f}$ with $\left|\operatorname{supp}\left(\mu_{1}\right)\right|<\left|\operatorname{supp}\left(\mu_{2}\right)\right|$. From the definition of a crisp greedoid, there exists $x \in \operatorname{supp}\left(\mu_{2}\right)-\operatorname{supp}\left(\mu_{1}\right)$ such that $\operatorname{supp}\left(\mu_{1}\right) \cup x \in \mathcal{F}$. Let $\mu=\mu_{1} \vee \sigma_{x}^{w(x)}$. It is clear that $\mu_{1}<\mu \leq \mu_{1} \vee \mu_{2}$. Also, since $m(\mu)=m\left(\mu_{1}\right)$ or $m(\mu)=w(x) \geq m\left(\mu_{2}\right), \mathcal{F}_{f}$ satisfies (F2). Thus $\mathfrak{G}$ is a fuzzy greedoid.

With this proposition, it becomes easy to generate a large number of classes of examples of fuzzy greedoids. We give two such classes.

\section{Example 1. (Weighted Branching Fuzzy Greedoids)}

Suppose that $\Gamma$ is an undirected graph and $\rho \in V(\Gamma)$ is a vertex. The set $\mathcal{F}$ of all edge sets of trees in $\Gamma$ which contain $\rho$ is the collection of feasible sets for the undirected branching greedoid $G\left(\Gamma_{\rho}\right)$ of $\Gamma$ with root $\rho$. Therefore, if $w: E \rightarrow[0,1]$ is a weight function, then $\mathfrak{G}\left(\Gamma_{\rho}, w\right)$ is the weighted undirected branching fuzzy greedoid of $\Gamma$ with root $\rho$ and weight function $w$. If $\Gamma$ is a directed graph, by using arborescences rooted at $\rho$, we obtain the weighted directed branching fuzzy greedoid of $\Gamma$ with root $\rho$ and weight function $w$.

In particular, if $\Gamma$ is the following graph

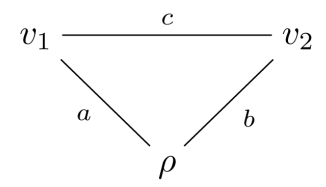

and $w: E \rightarrow[0,1]$ is defined by $w(a)=3 / 4, w(b)=1, w(c)=1 / 2$, then the following fuzzy set system is the feasible fuzzy sets of $\mathfrak{G}\left(\Gamma_{\rho}, w\right)$. (We use $\left(h_{1}, h_{2}, h_{3}\right)$ to denote the fuzzy set $\sigma_{a}^{h_{1}} \vee \sigma_{b}^{h_{2}} \vee \sigma_{c}^{h_{3}}$.)

$$
\mathcal{F}_{f}=\left\{(0,0,0),\left(\frac{3}{4}, 0,0\right),(0,1,0),\left(\frac{3}{4}, 1,0\right),\left(\frac{3}{4}, 0, \frac{1}{2}\right),\left(0,1, \frac{1}{2}\right)\right\} .
$$

\section{Example 2. (Weighted Poset Greedoids)}

Suppose $P$ is a finite poset. The collection of ideals of $P$ form the feasible sets for the poset greedoid $G(P)$ of $P$. Let $w: P \rightarrow[0,1]$ be a weight function, then by the previous proposition, $\mathfrak{G}(P, w)$ is the weighted poset fuzzy greedoid of $P$.

In particular, if $P$ has the following Hasse diagram.

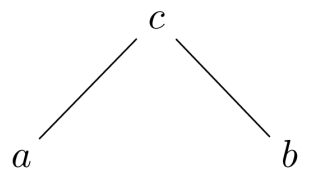

If $w(a)=1 / 2, w(b)=1 / 3, w(c)=3 / 8$, then the following fuzzy set system if the feasible fuzzy set of 
$\mathfrak{G}(P, w)$.

$$
\mathcal{F}_{f}=\left\{(0,0,0),\left(\frac{1}{2}, 0,0\right),\left(0, \frac{1}{3}, 0\right),\left(\frac{1}{2}, \frac{1}{3}, 0\right),\left(\frac{1}{2}, \frac{1}{3}, \frac{3}{8}\right)\right\}
$$

Although weighted greedoids give a large number of examples of fuzzy greedoids, not every fuzzy greedoid can be defined as a weighted greedoid, as the following example shows:

Example 3. Let $E=\{a, b\}$ and for $n=2,3, \cdots$ define $\mu_{n}=((n-1) / n, 0)$ and $v_{n}=((n-1) / n, 1 / 2)$. If $\mathcal{F}_{f}=\left\{\mu_{n}, v_{n} \mid n=2,3, \cdots\right\} \cup\{(0,0)\}$ then it is easy to show that $\mathfrak{G}=\left(E, \mathcal{F}_{f}\right)$ is a fuzzy greedoid.

However, as weighted greedoids, by definition, must have a finite collection of feasible fuzzy sets, this fuzzy greedoid cannot be a weighted greedoid.

\section{Fuzzy Languages}

In addition to a set system of feasible sets, a crisp greedoid can be defined as an ordered language with given properties. By defining a fuzzy language on a finite set, a fuzzy greedoid can be defined in the same manner. In this section, we determine the conditions necessary for a fuzzy language to define a fuzzy greedoid.

We begin by defining a fuzzy language on a set $E$.

Definition 7. Suppose $E=\left\{e_{1}, \cdots, e_{n}\right\}$. A fuzzy word $\omega$ on $E$ is defined by

$$
\omega=\sigma_{e_{1}}^{h_{1}} \sigma_{e_{2}}^{h_{2}} \cdots \sigma_{e_{k}}^{h_{k}},
$$

where $h_{i}>0$ for $i=1, \cdots, k$. A fuzzy language $\mathcal{L}_{f}$ is a collection of fuzzy words. For every word $\omega \in \mathcal{L}_{f}$ there is a fuzzy set associated to it,

$$
\vee \omega=\sigma_{e_{1}}^{h_{1}} \vee \sigma_{e_{2}}^{h_{2}} \vee \cdots \vee \sigma_{e_{k}}^{h_{k}} .
$$

In a standard abuse of notation, we will use $\operatorname{supp}(\omega)$ to denote $\operatorname{supp}(\vee \omega)$. We will let 0 represent the empty word.

In order to show when a fuzzy language defines a fuzzy greedoid, we need to be able to go from a collection of fuzzy words to a collection of fuzzy sets, and vice versa. Suppose $\mathcal{L}_{f}$ is a fuzzy language. Define the corresponding collection of fuzzy sets $\mathcal{F}\left(\mathcal{L}_{f}\right)$ by $\mathcal{F}\left(\mathcal{L}_{f}\right)=\left\{\vee \omega \mid \omega \in \mathcal{L}_{f}\right\}$.

For the other direction, Suppose $\left(E, \mathcal{F}_{f}\right)$ is a fuzzy set system, with $\mu \in \mathcal{F}_{f}$. Let $\operatorname{supp}(\mu)=\left\{e_{1}, \cdots, e_{k}\right\}$ and $h_{i}=\mu\left(e_{i}\right)$. Define a feasible ordering $e_{1}, \cdots, e_{k}$, on $\mu$ (on $\left.\operatorname{supp}(\mu)\right)$ if $\sigma_{e_{1}}^{h_{1}} \vee \sigma_{e_{2}}^{h_{2}} \vee \cdots \vee \sigma_{e_{i}}^{h_{i}} \in \mathcal{F}_{f}$ for all $1 \leq i \leq k$. For any collection of fuzzy sets, define $\mathcal{L}\left(\mathcal{F}_{f}\right)$ to be the set of all feasible orderings of all fuzzy sets $\mu \in \mathcal{F}$.

Given this ability to switch from a fuzzy language to a fuzzy set system, we now consider the properties that the languages for crisp greedoids have. Mirroring the properties possessed by crisp greedoids, we obtain the following theorem.

Theorem 1. Suppose $E$ is a finite set and $\mathcal{L}_{f}$ is a fuzzy language on E. Then $\mathcal{F}\left(\mathcal{L}_{f}\right)$ forms the feasible fuzzy sets of a fuzzy greedoid if and only if

(L1) $0 \in \mathcal{L}_{f}$;

(L2) If $\omega_{1} \omega_{2} \in \mathcal{L}_{f}$ then $\omega_{1} \in \mathcal{L}_{f}$

(L3) If $\omega_{1}, \omega_{2} \in \mathcal{L}_{f}$ with $\left|\operatorname{supp}\left(\omega_{1}\right)\right|<\left|\operatorname{supp}\left(\omega_{2}\right)\right|$ then there exists $x \in \operatorname{supp}\left(\omega_{2}\right)-\operatorname{supp}\left(\omega_{1}\right)$ with $\omega_{1} \sigma_{x}^{h} \in \mathcal{L}_{f}$ where $h=\left(\vee \omega_{2}\right)(x)$.

Proof. We need to show that $\mathcal{F}_{f}=\mathcal{F}\left(\mathcal{L}_{f}\right)$ satisfies both (F1) and (F2). Let $\mu \in \mathcal{F}_{f}$. There exists $\omega \in \mathcal{L}_{f}$ such that $\mu=v \omega$. Suppose $\omega=\sigma_{e_{1}}^{h_{1}} \sigma_{e_{2}}^{h_{2}} \cdots \sigma_{e_{k}}^{h_{k}}$. By property (L2), $\omega-\sigma_{e_{k}}^{h_{k}} \in \mathcal{L}_{f}$. Thus $\vee\left(\omega-\sigma_{e_{k}}^{h_{k}}\right)=\mu-\sigma_{e_{k}}^{h_{k}} \in \mathcal{F}_{f}$. Thus $\mathcal{F}_{f}$ satisfies property (F1). 
Let $\mu_{1}, \mu_{2} \in \mathcal{F}_{f}$ with $0 \leq\left|\operatorname{supp}\left(\mu_{1}\right)\right|<\left|\operatorname{supp}\left(\mu_{2}\right)\right|$. Thus there exists $\omega_{1}, \omega_{2} \in \mathcal{L}_{f}$ with $\mu_{i}=\vee \omega_{i}$ and with $\left|\operatorname{supp}\left(\omega_{1}\right)\right|<\left|\operatorname{supp}\left(\omega_{2}\right)\right|$. By property (L3), there exists $x \in \operatorname{supp}\left(\omega_{2}-\omega_{1}\right)$ such that $\omega_{1} \sigma_{x}^{h} \in \mathcal{L}_{f}$ where $h=\left(\vee \omega_{2}\right)(x)$. Define $\omega=\vee\left(\omega_{1} \sigma_{x}^{h}\right)$. Thus $\mu_{1}<\mu \leq \mu_{1} \vee \mu_{2}$ and $m(\mu) \geq \min \left\{m\left(\mu_{1}\right), h\right\} \geq \min \left\{m\left(\mu_{1}\right), m\left(\mu_{2}\right)\right\}$.

Therefore $\mathcal{F}_{f}$ satisfies (F2) and so a language satisfying properties (L1), (L2), (L3) determines a greedoid.

All that is left to show is that $\mathcal{L}_{f}=\mathcal{L}\left(\mathcal{F}\left(\mathcal{L}_{f}\right)\right)$. Since $\mathcal{L}_{f}$ satisfies (L2), this is straightforward and is omitted.

Similarly to crisp greedoids, Theorem 1 gives another cryptomorphic definition of a fuzzy greedoid. Next, we consider some of the functions that also give cryptomorphic definitions of crisp greedoids and consider their fuzzy versions. The two most common such functions are the rank function and the rank closure operator. We begin with the rank function for a fuzzy greedoid.

\section{Rank Function}

As with crisp greedoids, there is a natural definition of a rank function for fuzzy sets originally given in [5].

Definition 8. If $\mathfrak{G}=\left(E, \mathcal{F}_{f}\right)$ is a fuzzy greedoid on $E$, then the rank function $r:[0,1]^{E} \rightarrow[0, \infty)$ is defined by $r(\mu)=\sup \left\{|\eta|: \eta \in \mathcal{F}_{f}, \eta \leq \mu\right\}$.

Notice that for any fuzzy set $\mu, 0^{E} \leq \mu$ and $0^{E} \in \mathcal{F}_{f}$, so $r(\mu) \geq 0$.

Although we cannot guarantee that for any fuzzy set $\mu$, there exists $\alpha \leq \mu$ with $\alpha \in \mathcal{F}_{f}$ and $|\alpha|=r(\mu)$, we can get arbitrarily close, as stated in the next lemma.

Lemma 9. If $\mathfrak{G}=\left(E, \mathcal{F}_{f}\right)$ is a fuzzy greedoid with rank function $r$ and $\mu \in[0,1]^{E}$, then for any $\epsilon>0$, there exists $\alpha \in \mathcal{F}_{f}$ with $\alpha \leq \mu$ and $r(\mu)-|\alpha| \leq \epsilon$.

We first consider the properties possessed by $r$. In crisp greedoids, the rank function $r$ is characterized by the following four properties (Theorem V.1.1 from [7]):

1) $r(\varnothing)=0$

2) $r(X) \leq|X|$ for any $X \subseteq E$;

3) If $X \subseteq Y$ then $r(X) \leq r(Y)$ for any $X, Y \subseteq E$;

4) If $X \subseteq E, x, y \in E, r(X)=r(X \bigcup x)=r(X \bigcup y)$, then $r(X)=r(X \bigcup\{x, y\})$.

The fuzzy equivalents of these four properties are:

(R1) $r\left(0^{E}\right)=0$;

(R2) If $\mu \subseteq[0,1]^{E}$, then $r(\mu) \leq|\mu|$;

(R3) If $\mu_{1}, \mu_{2} \subseteq[0,1]^{E}$ with $\mu_{1} \leq \mu_{2}$, then $r\left(\mu_{1}\right) \leq r\left(\mu_{2}\right)$;

(R4) If $\mu \in[0,1]^{E}, x, y \in E, h, k \in(0,1]$, and $r(\mu)=r\left(\mu \vee \sigma_{x}^{h}\right)=r\left(\mu \vee \sigma_{y}^{k}\right)$, then $r(\mu)=r\left(\mu \vee \sigma_{x}^{h} \vee \sigma_{y}^{k}\right)$.

The natural question is whether these properties still hold for fuzzy greedoids, and if they do, whether they characterize the rank function of a fuzzy greedoid.

Proposition 10. If $\mathfrak{G}=\left(E, \mathcal{F}_{f}\right)$ is a fuzzy greedoid with rank function $r:[0,1]^{E} \rightarrow[0, \infty)$ then $r$ satisfies properties (R1), (R2), (R3), and (R4).

Proof. (R1) is trivial. Since $\eta \leq \mu$ implies that $|\eta| \leq|\mu|$, (R2) follows automatically.

Suppose $\mu_{1}, \mu_{2} \in[0,1]^{E}$ with $\mu_{1} \leq \mu_{2}$. Let $\eta \leq \mu_{1}$ with $\eta \in \mathcal{F}_{f}$. Therefore $|\eta| \leq r\left(\mu_{2}\right)$. Thus $r\left(\mu_{1}\right) \leq r\left(\mu_{2}\right)$, proving property (R3). 
Suppose $\quad \mu \in[0,1]^{E}, \quad x, y \in E, \quad h, k \in(0,1] \quad$ with $\quad r\left(\mu \vee \sigma_{x}^{h} \vee \sigma_{y}^{k}\right)>r(\mu)$. Let $\quad 0<\epsilon_{1} \quad$ and $0<\epsilon_{2}<\frac{1}{2}\left(r\left(\mu \vee \sigma_{x}^{h} \vee \sigma_{y}^{k}\right)-r(\mu)\right)$. By Lemma 9, there exists $\alpha, \beta \in \mathcal{F}_{f}$ with $\alpha \leq \mu,|\alpha|>r(\mu)-\epsilon_{1}$ and $\beta \leq \mu \vee \sigma_{x}^{h} \vee \sigma_{y}^{k},|\beta|>r\left(\mu \vee \sigma_{x}^{h} \vee \sigma_{y}^{k}\right)-\epsilon_{2}$. Let $\omega_{\alpha}$ and $\omega_{\beta}$ be feasible orderings of $\alpha$ and $\beta$ respectively.

By choice of $\epsilon_{2}, \operatorname{supp}(\alpha) \subseteq \operatorname{supp}(\mu) \subset \operatorname{supp}(\beta)$, so property (L3) implies that there exists $z \in \operatorname{supp}(\beta)-\operatorname{supp}(\alpha)$ such that $\omega_{\alpha} \sigma_{z}^{\beta(z)} \in \mathcal{L}\left(\mathcal{F}_{f}\right)$. By choice of $\alpha$, this implies $z \in\{x, y\}$, and therefore $r\left(\mu \vee \sigma_{x}^{h}\right)>r(\mu)$ or $r\left(\mu \vee \sigma_{y}^{k}\right)>r(\mu)$. Thus property (R4) is satisfied.

Given any rank function $r:[0,1]^{E} \rightarrow[0, \infty)$, we define a fuzzy set system $\mathcal{F}_{f}$ as follows: $\mathcal{F}_{f}=\left\{\mu \in[0,1]^{E}|r(\mu)=| \mu \mid\right\}$. With Proposition 10, the next natural question is whether these four necessary properties are also sufficient for the rank function of a fuzzy greedoid. The answer to this is negative, as the following example shows.

Example 4. Let $E=\{a, b\}$. Define $r(\mu)=\left\{\begin{array}{ll}|\mu| / 2 & \text { if }|\mu|<\frac{1}{2} \\ |\mu| & \text { otherwise. }\end{array}\right.$ A quick check shows that (R1), (R2), (R3), and (R4) hold for this rank function. Consider $\mu$ defined by $\mu(a)=\mu(b)=1 / 4$. Since $r(\mu)=1 / 2=|\mu|$, $\mu \in \mathcal{F}_{f}$. If $\alpha \leq \mu$ with $|\operatorname{supp}(\alpha)|<2$, then $r(\alpha)=|\alpha| / 2<|\alpha|$. So $\alpha \notin \mathcal{F}_{f}$. Thus $\mathcal{F}_{f}$ does not satisfy (F1).

However, with the following additional property, we do obtain a fuzzy greedoid.

(R5) If $\mu \in[0,1]^{E}$ and $h \leq m(\mu)$, then for any $e \in E, r\left(\mu \vee \sigma_{e}^{h}\right)-r(\mu) \in\{0, h\}$.

Before showing that a function satisfying (R1)-(R5) is the rank function of a fuzzy greedoid, we shall need the following lemma:

Lemma 11. Suppose $r:[0,1]^{E} \rightarrow[0, \infty)$ satisfies properties (R1)-(R5) and

$$
\mathcal{F}_{f}=\left\{\mu \in[0,1]^{E}|r(\mu)=| \mu \mid\right\} \text {. }
$$

If $\mu \in \mathcal{F}_{f}$ and $e \in \operatorname{supp}(\mu)$ with $\mu(e)=m(\mu)$, then $\mu-\sigma_{e}^{m(\mu)} \in \mathcal{F}_{f}$.

Proof. Suppose $\operatorname{supp}(\mu)=\left\{e_{1}, \cdots, e_{k}\right\}$ with $\mu\left(e_{i}\right)=h_{i}$ and $h_{k} \leq h_{i}$ for all $i$. Let $\eta=\bigvee_{i-1}^{k-1} \sigma_{e_{i}}^{h_{i}}$.

If $r(\eta)=r(\mu)$, then using (R2),

$$
|\eta| \geq r(\eta)=r(\mu)=|\mu|=|\eta|+h_{k} .
$$

This is clearly a contradiction, so $r(\eta)<r(\mu)$. Since $h_{k} \leq m(\eta)$, properties (R2) and (R5) imply that

$$
|\eta|+h_{k}=r(\mu)=r\left(\eta \vee \sigma_{e_{k}}^{h_{k}}\right)=r(\eta)+h_{k} \leq|\eta|+h_{k} .
$$

Thus $|\eta|=r(\eta)$.

With this, we can now show:

Theorem 2. If a function $r:[0,1]^{E} \rightarrow[0, \infty)$ satisfies (R1)-(R5) then $r$ is a rank function for a fuzzy greedoid.

Proof. As above, let $\mathcal{F}_{f}=\left\{\mu \in[0,1]^{E}|r(\mu)=| \mu \mid\right\}$. We need to show that $\mathfrak{G}=\left(E, \mathcal{F}_{f}\right)$ satisfies both (F1) and (F2).

The previous lemma shows that if $\mu \in \mathcal{F}_{f}$ and $\mu(e)=m(\mu)$, then $\mu-\sigma_{e}^{m(\mu)} \in \mathcal{F}_{f}$. Therefore $\mathcal{F}_{f}$ 
satisfies (F1).

To show $\mathcal{F}_{f}$ satisfies (F2), we assume that for all $\mu_{1}, \mu_{2} \in \mathcal{F}_{f}$ with $\left|\operatorname{supp}\left(\mu_{1}\right)\right|<\left|\operatorname{supp}\left(\mu_{2}\right)\right|$, there is no such $\mu$ satisfying the requirements of (F2). Assume that $\mu_{1}$ and $\mu_{2}$ are a pair of fuzzy sets in $\mathcal{F}_{f}$ with $\left|\operatorname{supp}\left(\mu_{1}\right)\right|<\left|\operatorname{supp}\left(\mu_{2}\right)\right|$ and also with $\left|\operatorname{supp}\left(\mu_{1}\right) \cap \operatorname{supp}\left(\mu_{2}\right)\right|$ minimal. We now obtain a contradiction.

Let $a \in \operatorname{supp}\left(\mu_{1}\right)$ be such that $\mu_{1}(a)=m\left(\mu_{1}\right)$. If $a \in \operatorname{supp}\left(\mu_{1}\right) \cap \operatorname{supp}\left(\mu_{2}\right)$, then $\mu_{1}-\sigma_{a}^{\mu_{1}(a)} \in \mathcal{F}$ and

$$
\left|\operatorname{supp}\left(\mu_{1}-\sigma_{a}^{\mu_{1}(a)}\right) \cap \operatorname{supp}\left(\mu_{2}\right)\right|<\left|\operatorname{supp}\left(\mu_{1}\right) \cap \operatorname{supp}\left(\mu_{2}\right)\right|
$$

Contradicting the assumptions. Otherwise we obtain a pair $v_{1}=\mu_{1}-\sigma_{a}^{\mu_{1}(a)}, v_{2}=\mu_{2}$ feasible sets with $\left|\operatorname{supp}\left(v_{1}\right)\right|<\left|\operatorname{supp}\left(v_{2}\right)\right|, m\left(v_{1}\right) \geq m\left(\mu_{1}\right)$ and with $\left|\operatorname{supp}\left(v_{1}\right)-\operatorname{supp}\left(v_{2}\right)\right|<\left|\operatorname{supp}\left(\mu_{1}\right)-\operatorname{supp}\left(\mu_{2}\right)\right|$.

Continuing this process, we obtain a pair of feasible fuzzy sets $\eta_{1}, \eta_{2}$ with $\left|\operatorname{supp}\left(\eta_{1}\right) \cap \operatorname{supp}\left(\eta_{2}\right)\right|=\left|\operatorname{supp}\left(\mu_{1}\right) \cap \operatorname{supp}\left(\mu_{2}\right)\right|$ and with $\eta_{1}<\eta_{2}$ and $m\left(\eta_{1}\right) \geq m\left(\mu_{1}\right)$ and $m\left(\eta_{2}\right)=m\left(\mu_{2}\right)$. Thus, using (R4), it is straightforward to show that there exists

$b \in \operatorname{supp}\left(\eta_{2}\right)-\operatorname{supp}\left(\eta_{1}\right)=\operatorname{supp}\left(\mu_{2}\right)-\operatorname{supp}\left(\mu_{1}\right)$ with $\eta_{2} \vee \sigma_{b}^{\eta_{2}(b)} \in \mathcal{F}_{f}$. This contradicts the assumptions, thus showing that $\mathcal{F}_{f}$ satisfies (F2).

Regretfully, not every rank function satisfies (R5), as the following example shows:

Example 5. For the following weighted rooted graph (rooted at $\bullet$, weights listed on the outside of each edge), consider its weighted undirected branching fuzzy greedoid.

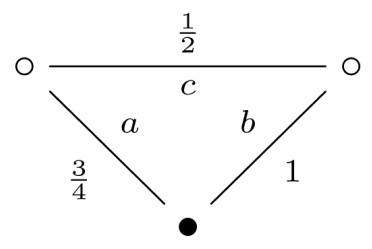

Notice that $\mu=\left(\frac{3}{4}, 0,0\right) \in \mathcal{F}$ and $\left(\frac{3}{4}, 0, \frac{1}{2}\right) \in \mathcal{F}$. Yet $r\left(\mu \vee \sigma_{c}^{\frac{3}{4}}\right)=\frac{5}{4} \neq r(\mu)+\frac{3}{4}$. Therefore this fuzzy greedoid does not satisfy (R5).

This leads to the natural question:

Question 1. Determine necessary and sufficient conditions for a function to be a rank function for a fuzzy greedoid.

To finish this section, we will obtain a partial answer to the above question. We will now characterize the properties of a rank function for a weighted greedoid. From the work above, the rank function of a weighted greedoid clearly satisfies properties (R1)-(R4). It is easy to see that such a rank function also satisfies

(R5') For all $e \in E$, there exists $h_{e} \in(0,1]$ such that for all $\mu \in[0,1]^{E}$, with $r(\mu)=|\mu|$, $r(\mu)=\sum_{e \in \operatorname{supp}(\mu)} h_{e}$.

All that remains is to show that a function satisfying properties (R1)-(R4) and (R5') defines a weighted greedoid. We first show that it must define a fuzzy greedoid.

Lemma 12. If a function $r:[0,1]^{E} \rightarrow[0, \infty)$ satisfies (R1)-(R4) and (R5'), then $r$ is a rank function for a fuzzy greedoid.

Proof. Once again, define $\mathcal{F}_{f}=\left\{\mu \in[0,1]^{E}|r(\mu)=| \mu \mid\right\}$. We need to show that $\mathfrak{G}=\left(E, \mathcal{F}_{f}\right)$ satisfies (F1) and (F2).

Suppose that $\mu \in[0,1]^{E}$. Since $E$ is finite, by (R5'), if $r(\mu)=r$, then there exists $\alpha \leq \mu$ with $|\alpha|=r$. Also, if $\mu \in \mathcal{F}_{f}$ and $e \in \operatorname{supp}(\mu)$, then $r\left(\mu-\sigma_{e}^{h_{e}}\right)<r(\mu)$, otherwise, (R2) would be contradicted. 
To show that $\mathcal{F}_{f}$ satisfies (F1), suppose $\mu \in \mathcal{F}_{f}$. For each $e \in \operatorname{supp}(\mu)$, let $S_{e}=r(\mu)-r\left(\mu-\sigma_{e}^{h_{e}}\right)$. Let $f \in \operatorname{supp}(\mu)$ be such that $S_{f}$ is minimal. If $\left|\mu-\sigma_{f}^{h_{f}}\right|=r\left(\mu-\sigma_{f}^{h_{f}}\right)$ then (F1) is satisfied, so assume that $\left|\mu-\sigma_{f}^{h_{f}}\right|>r\left(\mu-\sigma_{f}^{h_{f}}\right)$. Let $\alpha<\mu-\sigma_{f}^{h_{f}}$ be such that $|\alpha|=r(\alpha)=r\left(\mu-\sigma_{f}^{h_{f}}\right)$. Assume $\operatorname{supp}(\mu)-\operatorname{supp}(\alpha)=\left\{f, e_{1}, e_{2}, \cdots, e_{k}\right\}$.

If $r\left(\alpha \vee \sigma_{f}^{h_{f}}\right)>r(\alpha)$, then $r\left(\mu-\sigma_{e_{1}}^{h_{e_{1}}}\right) \geq r\left(\alpha \vee \sigma_{f}^{h_{f}}\right)>r\left(\mu-\sigma_{f}^{h_{f}}\right)$, contradicting the assumption about $f$. Therefore $r\left(\alpha \vee \sigma_{f}^{h_{f}}\right)=r(\alpha)$. For any $e_{i} \in\left\{e_{1}, \cdots, e_{k}\right\}, \alpha<\alpha \vee \sigma_{e_{i}}^{h_{e_{i}}} \leq \mu-\sigma_{f}^{h_{f}}$. Therefore $r(\alpha)=r\left(\alpha \vee \sigma_{e_{i}}^{h_{e_{i}}}\right)$. These facts, along with (R4) implies that $r(\alpha)=r(\mu)$, which contradicts the definition of $\alpha$. Thus $\alpha=\mu-\sigma_{f}^{h_{f}}$. Therefore $\mu-\sigma_{f}^{h_{f}} \in \mathcal{F}_{f}$, showing that $\mathfrak{G}$ satisfies (F1).

The proof to show that $\mathfrak{G}$ satisfies (F2) follows the exact steps of the proof of the previous theorem, however, instead of choosing elements $a$ with $\mu_{1}(a)$ minimal, at each step, choose an element $a$ with $r\left(\mu_{1}\right)-r\left(\mu_{1}-\sigma_{a}^{h_{a}}\right)$ minimal.

Theorem 3. Suppose that $r:[0,1]^{E} \rightarrow[0, \infty)$ satisfies $(R 1)-(R 4)$ and $\left(R 5^{\prime}\right)$, then $r$ is a rank function for a weighted greedoid.

Proof. As above, let $\mathcal{F}_{f}=\left\{\mu \in[0,1]^{E}|r(\mu)=| \mu \mid\right\}$, and let $\mathfrak{G}=\left(E, \mathcal{F}_{f}\right)$. The previous lemma shows that $\mathfrak{G}$ is a fuzzy greedoid. The collection of supports of feasible fuzzy sets forms the collection of feasible sets in a greedoid. For each element $e \in E$, define $w(e)=h_{e}$. It is straightforward to show that $\mathcal{F}_{f}$ is the collection of feasible fuzzy sets determined by the weights on the greedoid.

\section{Rank Closure}

We want to end with an example of the difference between crisp and fuzzy greedoids. In addition to feasible sets, ordered languages, and rank functions, crisp greedoids can be characterized by a closure operator. We will now show how the natural extension of the rank closure operator to fuzzy greedoids loses properties which would give a characterization of fuzzy greedoids. The natural extension is stated below:

Definition 13. Let $\mu \in[0,1]^{E}$. For each $x \in E$, define $\operatorname{cl}_{\mu}(x)=\sup \left\{h \mid r\left(\mu \vee \sigma_{x}^{h}\right)=r(\mu)\right\}$. With this, define the closure of $\mu, \operatorname{cl}(\mu)$ by $\operatorname{cl}(\mu)=\bigvee_{e \in E} \sigma_{e}^{\mathrm{cl}_{\mu}(e)}$.

For crisp greedoids, the rank of a subset and the rank of the closure of the subset are equal. This property leads to a collection of properties which can be used to characterize the closure operator of a crisp greedoid. Regretfully, this property no longer holds for fuzzy greedoids, as the following example shows.

Example 6. Consider the weighted branching greedoid on the following weighted graph:

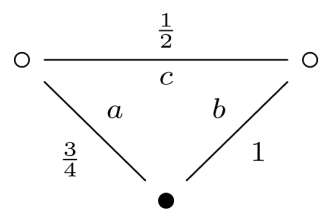

Notice that $r\left(0^{E}\right)=0$. For each $h<3 / 4, r\left(0^{E} \vee \sigma_{a}^{h}\right)=0$. Thus $\sigma_{a}^{3 / 4} \leq \mathrm{cl}\left(0^{E}\right)$, implies $r\left(\operatorname{cl}\left(0^{E}\right)\right) \geq 3 / 4$ (in fact $\left.r\left(\operatorname{cl}\left(0^{E}\right)\right)=7 / 4\right)$.

\section{Additional Research Areas}

As fuzzy greedoids are a new area of interest, there are a lot of directions research can go to understand these 
objects. Most of these areas come directly from the crisp greedoid definitions. We now mention four such areas of investigation for fuzzy greedoids.

1) The poset greedoid mentioned in Section 2 is actually an example of a combinatorial structure called an antimatroid (see [7] for an introduction to antimatroids). The weighted version leads to a definition of a fuzzy antimatroid as follows:

Definition 14. Given a finite set $E$ and a collection $\mathcal{F}_{f}$ of fuzzy sets on $E$, the fuzzy set system $\mathfrak{A}=\left(E, \mathcal{F}_{f}\right)$ is called a fuzzy antimatroid if

a) For every $\mu \in \mathcal{F}_{f}$ with $\mu \neq 0^{E}$, there exists $x \in \operatorname{supp}(\mu)$ such that $\mu-\sigma_{x}^{\mu(x)} \in \mathcal{F}_{f}$.

b) If $\mu_{1}, \mu_{2} \in \mathcal{F}_{f}$, then $\mu_{1} \vee \mu_{2} \in \mathcal{F}_{f}$.

A lot of work has been done in characterizing antimatroids in terms of a closure operator, shelling processes, etc. Determine the fuzzy versions of these properties for antimatroids.

2) In 1989 Gordon and McMahon [8] introduced a characteristic polynomial for greedoids. This polynomial involved summing terms over all subsets of the ground set of the greedoid. Determine a fuzzy equivalent for the characteristic polynomial for fuzzy greedoids which extends the characteristic polynomial introduced by Gordon and McMahon.

3) In addition to the rank closure introduced here, greedoids possess another closure operator, the kernel closure. If $G=(E, \mathcal{F})$ is a crisp greedoid, then the kernel closure, $\lambda$, of a subset $X$ is defined as $\lambda(X)=\{F \in \mathcal{F}: r(X \cup F)=r(X)\}$. Determine a fuzzy equivalent of the kernel closure and discover the properties that this closure possesses.

4) In addition to matroids and antimatroids, there are many classes of greedoids which have been studied extensively. In [5], Al-Hawary determined a fuzzy version for interval greedoids. There are also local poset greedoids, local forest greedoids, polymatroid, etc. (see [7] for definitions of these and other classes of greedoids). Determine fuzzy greedoid versions of these classes of greedoids, and determine which additional properties these classes of fuzzy greedoids must possess.

\section{Acknowledgements}

This research was supported by Summer Research Grants offered through Misericordia University.

\section{References}

[1] Zadeh, L.A. (1965) Fuzzy Sets. Information and Control, 8, 338-353. http://dx.doi.org/10.1016/S0019-9958(65)90241-X

[2] Goetschel Jr., R. and Voxman, W. (1988) Fuzzy Matroids. Fuzzy Sets and Systems, 27, 291-301. http://dx.doi.org/10.1016/0165-0114(88)90055-3

[3] Goetschel Jr., R. and Voxman, W. (1991) Fuzzy Rank Functions. Fuzzy Sets and Systems, 42, 245-258. http://dx.doi.org/10.1016/0165-0114(91)90150-O

[4] Goetschel Jr., R. and Voxman, W. (1992) Spanning Properties for Fuzzy Matroids. Fuzzy Sets and Systems, 51, $313-$ 321. http://dx.doi.org/10.1016/0165-0114(92)90022-V

[5] Al-Hawary, T. (2011) Fuzzy Greedoids. International Journal of Pure and Applied Mathematics, 70, $285-295$.

[6] Korte, B. and Lovász, L. (1981) Mathematical Structures Underlying Greedy Algorithms. Fundamentals of Computation Theory. Lecture Notes in Computer Sciences, 117, 205-209. http://dx.doi.org/10.1007/3-540-10854-8 22

[7] Korte, B., Lovász, L. and Schrader, R. (1991) Greedoids. Springer-Verlag, New York. http://dx.doi.org/10.1007/978-3-642-58191-5

[8] Gordon, G. and McMahon, E. (1989) A Greedoid Polynomial Which Distinguishes Rooted Arborescences. Proceedings of the American Mathematical Society, 107, 287-298. http://dx.doi.org/10.1090/s0002-9939-1989-0967486-0 